\title{
Mandibular defect reconstruction utilizing preoperative 3D modeling, plate pre-bending and a novel triad graft: a case report
}

\author{
Jon D. Wagner, Jeffrey P. Wagner, Stephen A. Wagner \\ Albuquerque Plastic and Maxillofacial Surgery, Albuquerque, NM 87109, USA.
}

Correspondence to: Dr. Jon D. Wagner, Albuquerque Plastic and Maxillofacial Surgery, 801 Encino Pl NE, Suite A3, Albuquerque, NM 87109, USA. E-mail: wagner.jon00@gmail.com

How to cite this article: Wagner JD, Wagner JP, Wagner SA. Mandibular defect reconstruction utilizing preoperative 3D modeling, plate pre-bending and a novel triad graft: a case report. Stomatological Dis Sci 2017;1:97-102.

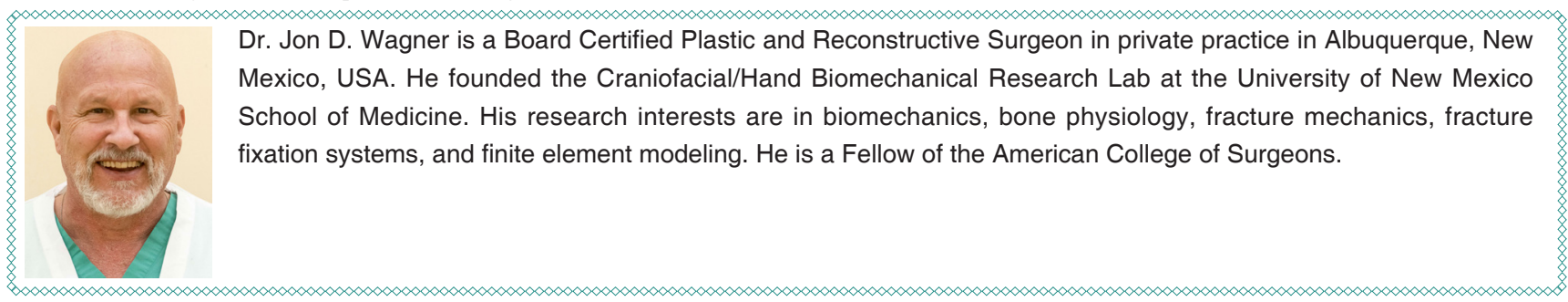

Article history:

Received: 30 Dec 2016

Accepted: 7 Apr 2017

Published: 29 Sep 2017

\section{Key words:}

3-dimensional prototyping,

osteoprotective membrane,

acellular dermal matrix,

demineralized bone matrix putty,

bone morphogenetic proteins,

osteopromotion,

pre-bending,

reconstruction plate

\begin{abstract}
The reconstruction of boney defects of the mandible are a challenge in surgical therapy despite advances in bone grafting. This case study details a 30 -year-old female who sustained a gunshot wound to the mandible leaving a $5 \mathrm{~cm}$ boney defect. This defect was reconstructed utilizing preoperative three-dimensional (3D) planning, an accurately pre-bent reconstruction plate, and a novel "triad" construct of autogenous bone graft, demineralized bone matrix, and an osteoprotective membrane. This reconstruction workflow allowed maintenance of the contour of the mandible and bone regeneration while also acting as a barrier to other tissues. This new technique reduced operative donor site morbidity and decreased surgical time dramatically, allowing the patient to be discharged the same day. The patient has a 5-year follow-up with panorex film and a biopsy of the graft area showing normal cortical bone. This case report illustrates the combination of advanced technology and novel products which can decrease operating time, decrease anesthesia time, increase precision, and most importantly decrease patient morbidity.
\end{abstract}

\section{INTRODUCTION}

Reconstruction of the mandible remains a challenge in surgical therapy despite advances in bone grafting. ${ }^{[1]}$ Current grafting techniques to repair discontinuity defects of the mandible include the following: non-

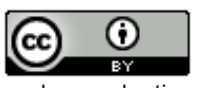
identical terms

This is an open access article licensed under the terms of Creative Commons Attribution 4.0 International License (https://creativecommons.org/licenses/by/4.0/), which permits unrestricted use, distribution,

For reprints contact: service@oaepublish.com

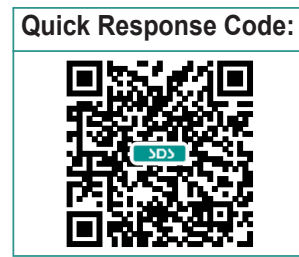


vascularized bone grafts using either autogenous bone or allografts or a combination of both; ${ }^{[2]}$ osteomyocutaneous pedicle transfer, ${ }^{[3]}$ ceramic grafts, ${ }^{[4]}$ and free tissue transfer. ${ }^{[5]}$ The most commonly used donor site is the iliac crest. ${ }^{\left[{ }^{6]}\right.}$ However, there may be significant morbidity including difficulties in walking, chronic pain, infection or fracture associated with large volume autografts from the iliac crest. ${ }^{[7]}$

The disadvantages of large volume autogenous grafting procedures have fostered a continuous search for alternative methods to promote regeneration of bone. ${ }^{\left[{ }^{[8]}\right.}$ As a consequence demineralized bone matrix $(\mathrm{DBM})^{[9]}$ and osteoprotective/osteopromotive membranes acellular dermal matrix $(A D M)^{[10]}$ were developed independently to promote bone regeneration and prevent scar tissue from invading the defect. The purpose of this report is to present a case where a "triad graft" composed of autogenous bone marrow, DBM, and ADM proved to be highly effective for the reconstruction of a mandibular defect caused by a gunshot. This new reconstructive procedure greatly reduces donor site morbidity, shortens operative time and may be performed as an outpatient procedure.

CARE critical appraisal guidelines were utilized for the description of this case report. This guideline is to provide a framework that supports transparency and accuracy in the publication of this case report. ${ }^{[11]} A$ timeline of this case report is provided in table form [Table 1].

\section{CASE REPORT}

The patient is a 30-year-old white female who incurred a gunshot wound to the face. The wound was caused by a low-velocity handgun projectile that entered lateral to the left oral commissure, caused a severely comminuted fracture of the mandibular angle, and remained lodged in the posterior cervical musculature. The patient was brought to a level 1 university hospital. After admission to the hospital, the patient was stabilized medically, and a tracheostomy was performed. Erich arch bars were secured to the teeth with circumdental wires. The occlusion was established with rigid intermaxillary fixation, and the mandibular wound was washed out and debrided extra-orally.

Over the following week, the wound underwent several washouts and debridements, however, an acute wound infection developed. The infection was cultured and treated with multiple additional debridements and intravenous antibiotics as medical therapy. The infection appeared to have resolved, and the patient was discharged in rigid intermaxillary fixation after 10 days in the hospital. However, a localized wound infection was detected during followup examinations. This infection was treated with incision and drainage, cultures, wound debridement and oral antibiotic therapy. The infection resolved completely after this treatment. The patient was placed in elastic intermaxillary fixation 1 month after discharge.

Table 1: Case report timeline

\begin{tabular}{lll}
\hline Date & Operation No. & Event \\
\hline Day 1 & Emergency room triage \\
& Tracheostomy \\
& Maxillary/mandibular fixation \\
& Conservative debridement \\
Day 10 & Patient discharged from hospital in rigid maxillary/mandibular fixation \\
Day 30 & Removal of rigid maxillary/mandibular fixation \\
& Placement of elastic maxillary/mandibular fixation \\
Day 120 & Computed tomography radiograph \\
& Three-dimensional model fabrication \\
& Model surgery \\
& Pre-bending of reconstruction template/reconstruction plate \\
& Measure screw length \\
& Outpatient surgery \\
Day 121 & Left coronoidectomy \\
& Maxillary/mandibular fixation \\
& Excision of cicatrix \\
& Rotation and alignment of proximal segment with pre-bent template/plate \\
& "Triad graft" \\
& Bicortical screw placement and securing of reconstruction plate \\
& Extraction of tooth \#18 \\
& Open bone biopsy of grafted bone segment left mandible \\
\hline
\end{tabular}




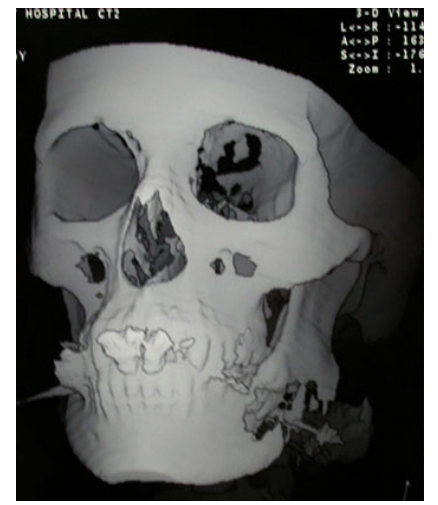

Figure 1: Three-dimensional computed tomography image of the maxillofacial skeleton that shows the discontinuity defect of the left mandibular angle

One month later the Erich arch bars were removed. During follow-up visits, the patient appeared to have ideal centric occlusion. However, a series of radiographs made during these visits showed a nonunion of the mandible and indicated that the proximal segment had rotated superiorly. The patient returned for outpatient reconstructive surgery four months after her initial discharge. Computed tomography images (Siemens SOMATOM Sensation Multi-slice, Seimens Healthliner, USA) of the mandible were obtained at $1.5 \mathrm{~mm}$ intervals on the day prior to surgery [Figure 1]. Data from these images were used to create a stereolithographic model of the mandible using an in-house rapid 3D printer (Z Corporation 20 North Ave. Burlington, MA) using available software (Mimics 7.3, Materialise, Ann Arbor, MI).

The data were transformed using software for interactive segmentation of the images into a format compatible with the 3D printer which produced a model of the maxillofacial region within $3 \mathrm{~h}$. Preoperative model surgery was then performed to rotate the proximal segment inferiorly into its correct position anatomically, and the proximal segment was secured in place with orange sticky wax (Keystone Industries, 480 Democrat Rd, Gibbstown, NJ). A universal mandibular reconstruction plate (Stryker Leibinger 4100 E. Milham Ave. Kalamazoo MI 49001) was then accurately and precisely bent to fit the contours of the $5 \mathrm{~cm}$ defect preoperatively ${ }^{[12]}$ [Figure 2]. In addition, screw length measurements were also determined in millimeters with the aid of a Boley gauge (Keystone, Orthozone.com) and recorded.

The following day, the patient underwent elective general nasotracheal anesthesia. A coronoidectomy was completed through an intra-oral incision to facilitate rotation of the proximal segment inferiorly, and the patient was placed into intermaxillary fixation with centric occlusion utilizing Erich arch bars. The

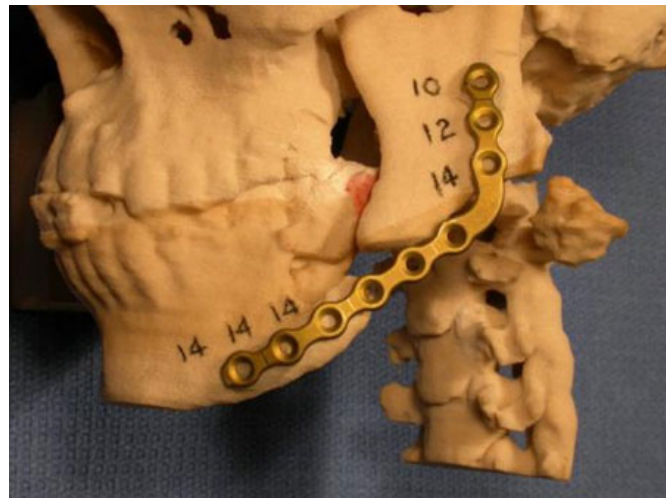

Figure 2: Photograph of a stereolithographic model of the craniofacial region that demonstrates the pre-bent reconstruction plate fixed to the mandibular segments after model surgery was performed. The numbers indicate the lengths of the screws to be used in millimeters

surgical wound intraorally did not communicate with the proposed reconstruction site. After re-draping and re-prepping of the extra-oral surgical site, the discontinuity defect was approached extra-orally via a Risden incision ${ }^{[13]}$ at the left mandibular angle. The wound cicatrix was excised, and the opposing surfaces of the proximal and distal segments were decorticated. Adhesions were dissected to allow rotation of the proximal mandibular segment inferiorly.

Simultaneously, the assistant surgeon, in a second sterile field, created a $2.5 \mathrm{~cm}$ incision was made over the anterior iliac crest as an assistant retracted the skin medially. This retraction allows the healed surgical scar to be located lateral to the crest for comfort, and decreases the risk for trauma to the lateral femoral cutaneous nerve. Cancellous bone was harvested through a $1-\mathrm{cm}$ aperture in diameter in the cortical bone from the anterior iliac crest as described by Wagner and Moore. ${ }^{[14]}$ There was $4 \mathrm{~mL}$ cancellous bone obtained.

The pre-bent mandibular reconstruction plate was used as a template to align the proximal and distal segments and was fixed to both segments with six 2.7-mm diameter bicortical screws. A sheet of ADM, $3 \mathrm{~cm} \times 6 \mathrm{~cm}$ (Alloderm ${ }^{\circledR}$ LifeCell Corporation, One Millennium Way, Branchburg, New Jersey) was meshed at a ratio of 1.5:1 (Zimmer 7701 Skin Graft Mesher, 2720 Broadbent Pkwy NE \# D, Albuquerque, $\mathrm{NM}$ ) and then positioned between the lingual mucosa and the medial wall of the discontinuity defect [Figure 3]. The cancellous bone was mixed in a 1:1 ratio with DBM putty (Grafton ${ }^{\circledR}$ Osteotech Inc., 51 James Way Eatontown, NJ). The resulting paste was then packed into the mandibular defect, and the free edge of the ADM was passively placed around the reconstruction plate by wrapping the free margin of the ADM superiorly to complete the construct [Figure 4]. 


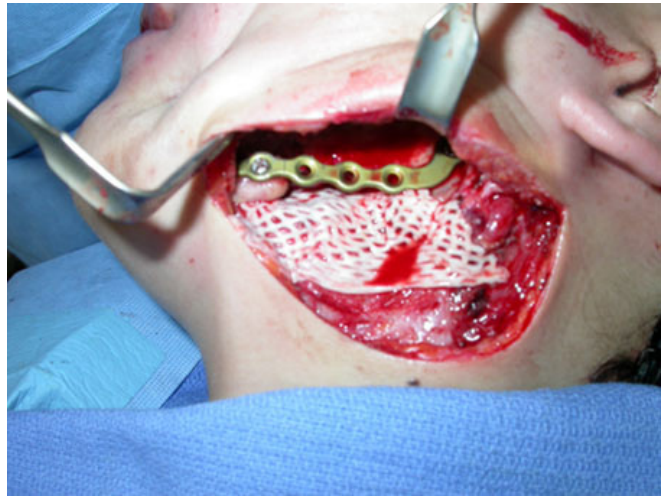

Figure 3: Intraoperative photograph of the placement of the acellular dermal matrix mesh between the oral mucosa and the medial aspect of the defect after fixation of the reconstruction plate

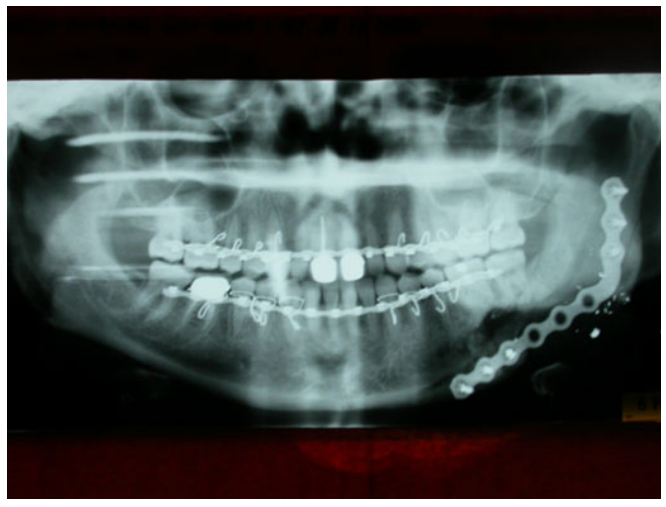

Figure 5: Postoperative panoramic radiograph of the maxillofacial region made four months after the reconstructive surgery

A 7-mm Jackson-Pratt flat silicone drain (Cardinal Health, 7000 Cardinal PI, Dublin, OH) was placed, the surgery was completed, the patient was placed in elastic fixation, and the patient was discharged the same day. The intermaxillary fixation was removed 2 weeks after surgery. A stable wound was noted on clinical examination, and the gradual consolidation of the autogenous cancellous bone-bone putty-dermal matrix "triad graft" was observed in radiographs made during follow-up visits [Figure 5]. She was lost to follow-up.

Five years following the patient's initial injury, the patient serendipitously presented to the emergency room complaining of pain in the left mandible in the region of mandibular left second molar (tooth \#18). On examination, tooth \#18 was deemed hopeless secondary to periodontal disease. The mandible was intact and stable. After the patient's consent and local anesthesia, tooth \#18 was removed without difficulty, and the consolidated graft of the mandible was inspected. The "pseudo-periosteum" was well demarcated and elevated with ease. A pre-planned bone biopsy of the consolidated graft was obtained. The patient's radiographs at this

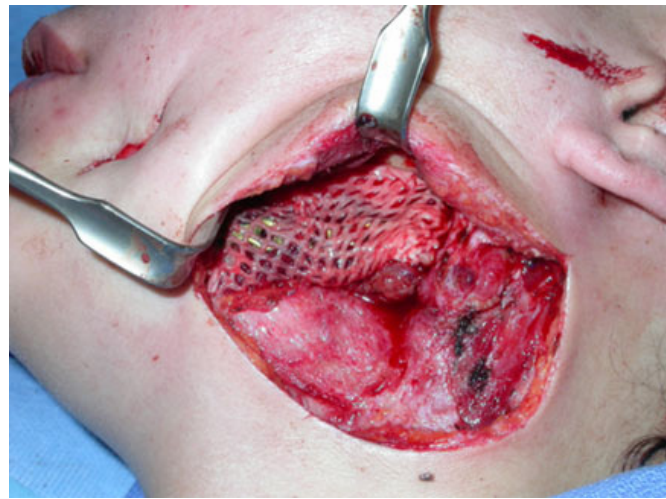

Figure 4: Intraoperative photograph of the "triad" graft composed of autogenous cancellous bone and demineralized bone matrix putty wrapped around the reconstruction plate with acellular dermis to fill the boney defect in the mandible

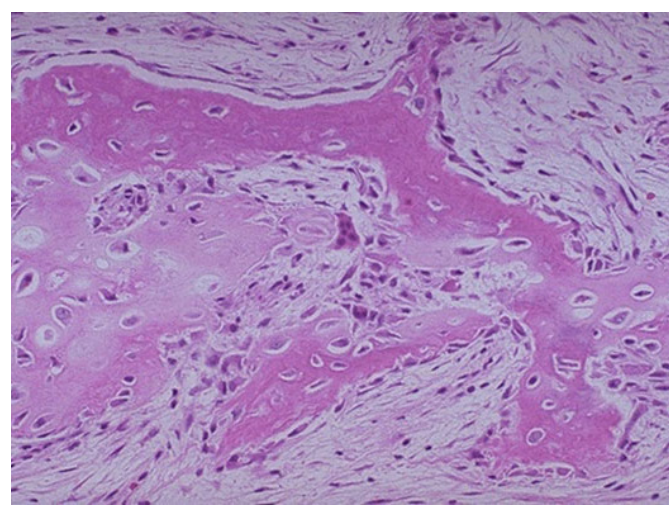

Figure 6: Bone biopsy obtained five years status post left mandibular reconstructive surgery. Histologic specimen demonstrates normal cortical bone with slight disorganization of the osteons. This is consistent with the previous injury and bone graft $(\mathrm{HE}, \times 400)$

time showed good incorporation of the graft and the histologic specimen obtained showed normal cortical bone with slight disorganization of the osteons [Figure 6] which is consistent with the previous injury and graft.

\section{DISCUSSION}

This case report describes the first use of a "triad" of graft materials to reconstruct a $5-\mathrm{cm}$ defect of the mandible caused by a gunshot. The patient had spent approximately 10 days in the hospital for multiple debridements of the wound and the treatment of a wound infection. Consequently, when the patient returned for the final stages of reconstructive surgery, it was important to use a procedure that minimized the length of stay in the hospital, minimized the morbidity associated with the surgery, and decreased the surgical time. Although the use of autogenous cancellous bone to promote regeneration was considered to be necessary, morbidity at the donor site also had to be minimized. 
For these reasons, we decided to harvest only a small amount of autogenous cancellous bone through a small aperture in the iliac crest. The autogenous graft was then mixed with DBM putty to provide a paste that would fill the defect, and also contains a high concentration of bone morphogenetic proteins. The paste was then wrapped in the meshed ADM that not only acted as an osteopromotive membrane, but also confined the paste to the contours of the defect. This logic led to the development of the "triad graft". Even though this triad combination has not been used previously, each of the components have been used individually and often in dual combination to promote bone regeneration. Each component plays a significant and somewhat different role in the promotion of bone regeneration.

Bone morphogenetic protein and bone-derived growth factors were first described by Urist et al. ${ }^{[15]}$ to induce bone formation. The extracellular matrix of bone contains a number of bone morphogenetic proteins (BMPs) that are required for bone formation. ${ }^{[16]}$ The most heavily studied bone morphogenetic proteins are BMP-2 and BMP-7. ${ }^{[17]}$ BMPs are currently being used to promote osteogenesis in a wide variety of clinical settings where bone growth is critical. Therefore, it is worthwhile to include autogenous cancellous bone, a rich source of BMPs, in bone graft implants. However, the collection of large amounts of autogenous cancellous bone causes significant morbidity as a consequence of extensive surgery at the donor site. Fortunately, DBM diminishes the need for large amounts of autogenous cancellous bone. ${ }^{[18]}$ Grafton $^{\circledR}$ is a proprietary form of DBM that retains growth factors and has been shown to induce new bone formation. ${ }^{[19,20]}$ DBM is a readily available graft material that can be processed to retain both osteoconductive and osteoinductive characteristics. ${ }^{[21]}$ DBM putty has been utilized successfully to reduce second site autogenous bone harvest sites in spinal surgery. ${ }^{[22]}$ Other often used DBMs are Allomatrix ${ }^{\circledR}$, Osteosponge ${ }^{\circledR}$, and Puros $^{\circledR}{ }^{[23]}$

Porous membranes have been used in periodontal and spinal surgery as mechanical barriers around bone defects since the early 1980s. ${ }^{[8,24]}$ These barriers create a secluded space around the defect to permit bone regeneration without the competition of other connective tissues. Among the membranes most extensively used are expanded polytetrafluoroethylene, polylactic acid, and polyglycolic acid. ADM was initially developed for use in the treatment of full thickness burns, and is a cadaveric dermal graft that has been decellularized to reduce the risk of rejection or inflammation. ${ }^{[25]}$ It acts as a scaffold for migration of host fibroblasts and facilitates attachment of surface epithelium. When used as a barrier, the basement membrane side of ADM prevents epithelial cell migration while the connective tissue side enhances cell repopulation and revascularization. When used for hard tissue regeneration applications, the ADM provides a matrix for the formation of neo-connective tissue. Perhaps the most important feature of ADM is that it does not have to be removed in a subsequent procedure. Other often used ADMs are SurgiMend ${ }^{\circledR}$, DermaCELL ${ }^{\circledR}$, and AlloMend ${ }^{\circledR}$.

There are 2 major concerns regarding the use of allografts: antigenicity, and the risk of disease transmission. DBM and ADMs have no immunological rejection as the antigenic surface structure of the bone is destroyed during demineralization by an acid environment, and the cellular component of the ADM is removed by a proprietary process. In regard to disease transmission, the probability of HIV transfer following appropriate demineralized bone allograft preparation has been calculated to be 1 in 2.8 billion. ${ }^{[26]}$

In conclusion, the major advantages of case reporting are the ability to make new observations, generate hypotheses, accumulate scientific data about rare disorders, do in-depth narrative studies, and serve as a major educational tool. The method is deficient mainly in being unable to deliver quantitative data. Nor can it prove cause-effect relationship or allow generalizations. Furthermore, there is a risk of overinterpretation, selection bias, publication bias, and positive outcome bias.

The surgical time for this case was reduced significantly $(>60 \%)$ as a product of the small aperture iliac graft harvesting and the accurate pre-bending of the definitive reconstruction plate utilizing 3D technology. It was possible to reduce the amount of autogenous cancellous bone required to promote healing since it was used in combination with DBM putty. The ADM acts as a barrier to fibrous ingrowth and appears to encourage consolidation of the boney construct. This approach greatly reduced donor site morbidity. Additional operative time was reduced by pre-bending the reconstruction plate, which provided a template for precise alignment of the mandibular segments and as a definitive plate fixation. This case report illustrates the combination of advanced technology and novel products which can decrease operating time, decrease anesthesia time, increase precision, and most importantly decrease patient morbidity.

\section{Authors' contributions}

Manuscript's preparation: J.D. Wagner

Manuscript's review: S.A. Wagner

Concept design: J.D. Wagner

Literature search: J.P. Wagner 


\section{Financial support and sponsorship None.}

\section{Conflicts of interest \\ There are no conflicts of interest.}

\section{Patient consent}

We discussed our operative plan with the patient. We also discussed the risks and potential complications, and obtained an informed/witnessed consent from the patient.

\section{Ethics approval}

According to the requirements of the affiliation, this case report does not require ethics approval.

\section{REFERENCES}

1. Marx RE. Mandibular reconstruction. J Oral Maxillofac Surg 1993;51:446-79.

2. Citardi MJ, Friedman CD. Nonvascularized autogenous bone grafts for craniofacial skeletal augmentation and replacement. Otolaryngol Clin North Am 1994;27:891-910.

3. Bell MS, Barron PT. The rib-pectoralis major osteomyocutaneous flap. Ann Plast Surg 1981;6:347-53.

4. Kent JN, Quinn JH, Zide MF, Guerra LR, Boyne PJ. Alveolar ridge augmentation using nonresorbable hydroxylapatite with or without autogenous cancellous bone. J Oral Maxillofac Surg 1983;41:629-42.

5. Hildalgo DA. Fibula free flap: a new method of mandible reconstruction. Plast Reconstr Surg 1989;84:71-9.

6. Burstein FD, Simms C, Cohen SR, Work F, Paschal M. Iliac crest bone graft harvesting techniques: a comparison. Plast Reconstr Surg 2000;105:34-9.

7. Silber JS, Anderson DG, Daffner SD, Brislin BT, Leland JM, Hilibrand AS, Vaccaro AR, Albert TJ. Donor site morbidity after anterior iliac crest bone harvest for single-level anterior cervical discectomy and fusion. Spine 2003;28:134-9.

8. Vaccaro AR, Chiba K, Heller JG, Patel TCh, Thalgott JS, Truumees E, Fischgrund JS, Craig MR, Berta SC, Wang JC; North American Spine Society for Contemporary Concepts in Spine Care. Bone grafting alternatives in spinal surgery. Spine $J$ 2002;2:206-15.

9. Ferretti C, Ripamonti U. Human segmental mandibular defects treated with naturally derived BMP's. J Craniofac Surg 2002;13:434-44.

10. Linde A, Hedner E. Recombinant bone morphogenetic protein-2 enhances bone healing, guided by osteopromotive e-PTFE membranes: an experimental study in rats. Calcif Tissue Int 1995;56:549-53.

11. CARE Case Report Guidelines. Seventh International Congress on Peer Review and Biomedical Publication September, 2013, Chicago, Illinois. Available from: http://www.care-statement.org. [Last accessed on May 2, 2017]

12. Wagner JD, Baack B, Brown GA, Kelly J. Rapid 3-dimensional prototyping for surgical repair of maxillofacial fractures: a technical note. J Oral Maxillofac Surg 2004;62:898-901.

13. Risdon FE. Ankylosis of the temporomandibular joint. $J$ Am Dent Assoc 1934;21:1933-7.

14. Wagner JD, Moore DL. The use of cylindrical osteotomes for harvesting cancellous bone from the ilium. J Oral Maxillofac Surg 1991;49:433-4.

15. Urist MR, Delange RJ, Finerman GA. Bone cell differentiation and growth factors. Science 1983;220:680-6.

16. Reddi AH. Regulation of cartilage and bone differentiation by BMP Curr Opin Cell Biol 1992;4:850-5.

17. Würzler KK, DeWeese TL, Sebald W, Reddi AH. Radiation-induced impairment of bone healing can be overcome by morphogenetic protein-2. J Craniofac Surg 1998;9:131-7.

18. Tilkeridis K, Touzopoulos P, Ververidis A, Christodoulou S, Kazakos $\mathrm{K}$, Drosos GI. Use of demineralized bone matrix in spinal fusion. World J Orthop 2014;5:30-7.

19. Edwards JT, Diegmann MH, Scarborough NL. Osteoinduction of human demineralized bone: characteristics in a rat model. Clin Orthop Relat Res 1998;(357):219-28.

20. Dang J, An H, Hilibrand A, Yoon ST, Kavanagh E, Boden S. Grafton and local bone have comparable outcomes to iliac crest bone in instrumented single-level lumbar fusions. Spine (Phila Pa 1976) 2012;37:1083-91

21. Strates BS, Teideman JJ. Contribution of osteoinductive and osteoconductive properties of demineralized bone matrix to skeletal repair. Eur J Exp Musculoskel Res 1993;2:61-7.

22. Sassard WR, Eidman DK, Gray PM, Block JE, Russo R, Russell JL, Taboada EM. Augmenting local bone with Grafton demineralized bone matrix for posterolateral lumbar spine fusion: avoiding second site autologous bone harvest. Orthopedics 2000;23:1059-64.

23. Snyder D, Browne T. Choosing bone graft substitutes: filling the gaps on evidence and pricing, webinar presentation. ECRI Institute [September 24, 2014]. Available from: https://www.ecri.org/ Documents/Webinar_PPT. [Last accessed on May 2, 2017]

24. Liu J, Kerns DG. Mechanisms of guided bone regeneration: a review. Open Dent J 2014;8:56-65.

25. Wainwright DJ. Use of an acellular allograft dermal matrix (AlloDerm) in the management of full-thickness burns. Burns 1995;21:243-8.

26. Nasr HF, Aichelmann-Reidy ME, Yukna RA. Bone and bone substitutes. Periodontol 2000 1999;19:74-86. 